\title{
PREVALÊNCTA DE ALTERAÇÕES PROUFERATIVAS GÁSTRICAS EM PACIENTES COM USO CRÔNICO DE INIBIDORES DE BOMBA DE PRÓTONS
}

\author{
Prevalence of gastric proliferative changes in patients with chronic use of proton pump inhibitor agents \\ Vivian de Souza MENEGASSI, Leticia Elizabeth Augustin CZECZKO, Larissa Santin Garcia CZECZKO, \\ Sergio Ossamu IOSHII, Julio Cesar PISANI, Odery RAMOS JUNIOR
}

Trabalho realizado no Serviço de Gastroenterologia e Endoscopia Digestiva do Hospital de Clínicas da Universidade Federal do Paraná, Curitiba, PR, Brasil.

DESCRITORES - Inibidores da bomba de prótons. Mucosa gástrica. Pólipos.
RESUMO - Racional - As medicações mais frequentemente prescritas e usadas de forma crônica por pacientes com queixas dispépticas pertencem ao grupo dos inibidores de bomba de prótons cujo principal representante é o omeprazol, utilizado clinicamente a cerca de 20 anos. Estudos recentes têm postulado a relação entre o uso crônico desta medicação e alterações proliferativas macroscópicas e microscópicas da mucosa do fundo e corpo gástrico, principalmente os pólipos de glândulas fúndicas. Objetivo - Analisar a frequência e o tipo de alterações proliferativas gástricas em usuários crônicos de inibidores de bomba de prótons e sua associação com idade, dose utilizada, tempo de uso, sintomatologia e infecção pelo Helicobacter pylori. Método - Estudo transversal de prevalência realizado no Serviço de Endoscopia Digestiva do Hospital de Clínicas da Universidade Federal do Paraná. Aplicado questionário com informações quanto ao uso destas drogas e realizada endoscopia digestiva alta em sujeitos em uso contínuo desta classe de medicamentos há pelo menos 12 meses. Realizadas biópsias de fundo, corpo e antro gástrico em todos os pacientes. Pólipos eram retirados ao serem identificados, para análise histopatológica. Realizada pesquisa do Helicobacter pylori por teste de urease e análise histopatológica. Resultados - Vinte e dois indivíduos foram incluídos sendo que seis $(27,3 \%)$ apresentaram alterações proliferativas da mucosa oxíntica. Destes, dois $(9,1 \%)$ apresentavam pólipos fúndicos esporádicos e quatro apresentavam exclusivamente alteração proliferativa microscópica como formação cística glandular. Houve significância estatística $(p<0,05)$ na associação entre idade $\geq 60$ anos e estas alterações. Não houve associação entre dose de uso, tempo de uso, sintomatologia e infecção pelo Helicobacter pylori. Conclusões - Ocorrem alterações proliferativas de mucosa oxíntica em indivíduos em uso crônico de inibidores de bomba de prótons. A associação entre idade e alterações proliferativas apresentou significância estatística.

\section{Correspondência:}

Vivian de Souza Menegassi,

e-mail: vimenegassi@yahoo.com.br

Fonte de financiamento: não há Conflito de interesses: não há

Recebido para publicação: 11/05/2010 Aceito para publicação: 14/07/2010

HEADINGS - Proton pump inhibitors. Gastric mucosa. Polyps.
ABSTRACT - Background - The medications most often prescribed and used chronically by patients with dyspeptic symptoms belong to the group of inhibitors of proton pump whose main representative is omeprazole, used clinically about 20 years. Recent studies have postulated the relationship between chronic use of this medication and macroscopic and microscopic proliferative changes in mucosal of gastric body, especially the fundic gland polyps. Aim - To analyze the frequency and type of gastric proliferative changes in chronic users of proton pump inhibitors proton and its association with age, dose, time of use, symptoms and Helicobacter pylori infection. Methods - A survey of prevalence was conducted in Digestive Endoscopy Service, Hospital de Clínicas, Federal University of Paraná, using questionnaire referring the use of these drugs and performed in subjects using this class of drugs during at least 12 months prior to the endoscopy. Gastric biopsies of fundus, body and antrum were performed in all patients. Polyps were removed when present for histopathological analysis. Research on Helicobacter pylori was done by urease test and histopathology. Results - Twenty-two individuals were included of which six (27.3\%) had proliferative changes of the mucosa. Of these, two (9.1\%) had sporadic fundic polyps and four only microscopic proliferative change as cystic glandular formation. Statistic significant ( $p<0.05$ ) association between age $\geq 60$ years and these changes was found. There was no association between dose of use, time of use, symptoms and Helicobacter pylori infection. Conclusions - It was observed the existence of proliferative mucosa changes in individuals with chronic use of inhibitors of proton pump. In this group, the association between age and proliferative changes was significant. 
INTRODUÇÃO

$\mathrm{O}$ $s$ inibidores de bomba de prótons (IBP's) são drogas amplamente utilizadas de forma crônica no nosso cotidiano e até hoje seu uso foi considerado inerte ao ser humano. Estudos recentes têm postulado a relação entre o uso crônico destas drogas com alterações proliferativas da mucosa do fundo e corpo gástrico ${ }^{2,3,6,8,10}$.

Controvérsias quanto a esta relação existem e já foram descritas em grandes estudos onde não houve diferença estatística entres os grupos. O tempo de tratamento com IBP's necessário para provocar alterações proliferativas gástricas ainda não foi determinado por ser bastante variável na literatura ${ }^{4}$.

Dentre as alterações proliferativas microscópicas estão a hiperplasia de células parietais, protusão de células parietais no lúmen de glândulas oxínticas e formações císticas glandulares ${ }^{11}$, enquanto as alterações macroscópica mais comums descritas são os pólipos fúndicos esporádicos os quais raramente apresentam achados de displasia9. Os indivíduos podem evoluir com sintomas como epigastralgia, azia e dispepsia ou mais comumente serem assintomáticos, podendo essas alterações serem achados de endoscopia 5 .

Este trabalho tem como objetivo determinar a prevalência de alterações proliferativas gástricas em pacientes que fazem uso crônico de IBP's, além de estudar se há associação entre os achados encontrados, considerando a idade do paciente; dose diária da droga; tempo de uso do medicamento; presença de sintomas dispépticos e infecção atual por Helicobacter pylori.

\section{MÉTODO}

Esse é um estudo transversal de prevalência realizado no Serviço de Gastroenterologia e Endoscopia Digestiva do Hospital de Clínicas da Universidade Federal do Paraná.

Foram avaliados todos os pacientes encaminhados com pedido para realização de exame endoscópico, no período de abril a setembro de 2009, que preencheram os seguintes critérios de inclusão: mais de 18 anos; em tratamento com IBP's há pelo menos 12 meses; compreensão e assinatura do Termo de Consentimento Livre e Esclarecido; pacientes que não tivessem história de alterações na coagulação e portadores de varizes no esôfago e/ou estômago, ou em uso de medicação anticoagulante e anti-inflamatórios.

A seleção dos pacientes foi realizada na sala de espera do serviço, enquanto esperavam para execução de suas endoscopias digestivas altas. Foram convidados a participar do estudo, os que preencheram os critérios de inclusão e deram seu consentimento escrito, responderam o questionário e posteriormente foram submetidos ao exame. O questionário visava identificar o nome, sexo, raça, idade, uso atual de inibidor de bomba de próton determinando tipo, dose, tempo e motivo da utilização, sintomas atuais, comorbidades e demais medicações em uso atual.

Os exames endoscópicos foram realizados utilizando-se aparelhos de videoendoscopia da marca Fujinon ${ }^{\circledR}$ Série 200 e Olympus ${ }^{\circledR}$ Evis CV-100.

Em todos os pacientes foram realizadas biópsias de dois pontos do fundo, corpo e antro gástrico, no caso de alterações macroscópicas como pólipos gástricos, que eram retirados e enviados para análise juntamente com as biópsias. O H. pylori foi pesquisado através do teste da urease e do estudo histopatológico.

\section{RESULTADOS}

Foram incluídos neste estudo 22 sujeitos que compareceram para realização de endoscopia digestiva alta, de maneira eletiva e previamente agendada, em uso crônico de inibidores de bomba de próton, no período de abril a outubro de 2009.

Dos sujeitos incluídos, 14 (63,6\%) eram do sexo feminino e pertenciam à faixa etária $<60$ anos. Quanto à etnia, 17 (77,3\%) eram brancos, três $(13,6 \%)$ pardos e dois $(9 \%)$ negros.

Dos 22 sujeitos do estudo, 13 (59\%) permaneciam com sintomas dispépticos, com queixas ocasionais de epigastralgia, azia e plenitude, apesar do uso contínuo de inibidores de bomba de prótons. Os demais sujeitos encontravam-se totalmente assintomáticos

Dezoito (81,8\%) apresentavam comorbidades sendo relatadas hipertensão arterial sistêmica, diabete melito, hipotireoidismo, artrose, lupus eritematoso sistêmico, angina estável, depressão e síndrome do intestino irritável. Todos faziam uso de medicações adequadas para seus diagnósticos.

As características gerais dos indivíduos do estudo são demonstradas na Tabela 1.

TABELA 1 - Características gerais dos 22 indivíduos estudados

\begin{tabular}{l|c|c|c|}
\hline Características & & $\mathbf{n}$ & $\%$ \\
\hline Sexo & Feminino & 14 & $(63,6 \%)$ \\
\hline \multirow{2}{*}{ Idade } & Masculino & 8 & $(36,4 \%)$ \\
\hline \multirow{2}{*}{ Etnia } & $<60$ anos & 14 & $(63,6 \%)$ \\
\hline & $\geq 60$ anos & 8 & $(36,4 \%)$ \\
\hline Sintomas & Branca & 17 & $(77,3 \%)$ \\
\hline dispépticos & Pardo & 3 & $(13,6 \%)$ \\
\hline & Negro & 2 & $(9 \%)$ \\
\hline Comorbidades & Presente & 13 & $(59 \%)$ \\
\hline & Ausente & 9 & $(41 \%)$ \\
\hline & Presente & 18 & $(81,8 \%)$ \\
\hline
\end{tabular}

Todos os participantes estavam em uso atual de inibidores de bomba de próton sendo que apenas três $(13,6 \%)$ participantes manipulavam as medicações. Vinte e um $(95,4 \%)$ faziam uso de omeprazol e apenas um referiu uso de pantoprazol. 
Questionando-se a dose utilizada atualmente, notou-se que $14(63,6 \%)$ indivíduos utilizavam 20mg/ dia e que oito $(36,4 \%) \geq 40 \mathrm{mg} / \mathrm{dia}$.

Para uma melhor avaliação do tempo total de uso destes medicamentos, dividiu-se a análise em dois intervalos de tempo considerando: uso < 24 meses e $\geq 24$ meses. Observou-se que $16(72,7 \%)$ participantes faziam uso $\geq 24$ meses e que seis $(27,3 \%)$ referiam uso $<24$ meses. O tempo mínimo de uso referido foi de três meses.

A dose atual e tempo de uso de inibidores de bomba de prótons pelos 22 indivíduos estudados estão descritos na Tabela 2.

TABELA 2 - Dose atual e tempo de uso de IBP's pelos 22 indivíduos estudados

\begin{tabular}{|c|c|c|c|}
\hline \multicolumn{2}{|c|}{ Inibidores de bomba de prótons } & $n$ & $\%$ \\
\hline \multirow[t]{2}{*}{ Dose atual } & $<40 \mathrm{mg} / \mathrm{dia}$ & 14 & $(63,6 \%)$ \\
\hline & $\geq 40 \mathrm{mg} / \mathrm{dia}$ & 8 & $(36,4 \%)$ \\
\hline \multirow[t]{2}{*}{ Tempo de uso } & $<24$ meses & 6 & $(27,3 \%)$ \\
\hline & $\geq 24$ meses & 16 & $(72,7 \%)$ \\
\hline
\end{tabular}

Quando interrogados sobre o diagnóstico que ocasionou o uso contínuo desta medicação, notou-se que alguns participantes apresentavam mais de uma condição associada. Os diagnósticos relatados foram doença do refluxo gastroesofágico, hérnia de hiato, gastrite e úlcera péptica.

De todos os exames endoscópicos realizados, seis $(27,3 \%)$ sujeitos apresentavam resultado normal. Outros achados endoscópicos foram hérnia de hiato por deslizamento, esofagite erosiva distal com graus A/ B/ D de Los Angeles, esôfago de Barrett, gastrite enantematosa de antro, úlceras gástricas de aspecto péptico em atividade e com aspecto cicatricial, duodenites erosiva e enantematosa.

A prevalência total de achados proliferativos macroscópicos e microscópicos da mucosa oxíntica foi de seis pacientes, $27,3 \%$ (IC-95\% = 10,7 a 50,2). Macroscopicamente, dois (9,1\% - IC-95\% = 1,1 a 29,2) indivíduos apresentavam pólipos $<5 \mathrm{~mm}$. A análise histopatológica confirmou 0 achado de pólipos fúndicos esporádicos. Já nas biópsias realizadas na procura de alterações proliferativas microscópicas, verificou-se que quatro $(18,2 \%$ - IC- $95 \%=5,2$ a 40,0$)$ indivíduos apresentavam exclusivamente formação cística glandular em corpo e/ou fundo. Um dos pacientes com pólipo apresentou simultaneamente formação cística glandular.

Outras alterações não proliferativas também foram evidenciadas no material biopsiado da mucosa oxíntica, como gastrite crônica em diferentes graus. A biópsia de mucosa antral também demonstrou alterações como gastrite crônica, gastrite atrófica com metaplasia intestinal e o achado de um adenocarcinoma moderadamente diferenciado invasor em úlcera de antro gástrico, de aspecto péptico ao exame endoscópico.

$O$ teste de urease apresentou-se positivo em somente três $(13,6 \%)$ pacientes. Entretanto a análise histopatológica para pesquisa de $H$. pylori demonstrou grau maior de infecção dos sujeitos estudados evidenciando oito $(36,4 \%)$ resultados positivos para sua presença.

Alterações endoscópicas e histopatológicas da mucosa oxíntica dos 22 indivíduos do grupo estão sumarizadas na Tabela 3.

TABELA 3 - Alterações endoscópicas e histopatológicas da mucosa oxíntica dos 22 indivíduos estudados

\begin{tabular}{l|c|c|c}
\hline Alterações endoscópicas e histopatológicas & n & $\%$ \\
\hline Alterações endoscópicas & Presente & 16 & $(63,7 \%)$ \\
\hline \multirow{3}{*}{ Alterações proliferativas } & Ausente & 6 & $(27,3 \%)$ \\
\hline \multirow{3}{*}{ Teste da Uréase } & Presente & 6 & $(27,3 \%)$ \\
\hline \multirow{2}{*}{ H. pylori na histopatologia } & Ausente & 16 & $(63,7 \%)$ \\
& Positivo & 3 & $(13,6 \%)$ \\
\hline & Negativo & 9 & $(86,4 \%)$ \\
\hline & Presente & 8 & $(36,4 \%)$ \\
\hline
\end{tabular}

H. pylori $=$ Helicobacter pylori

Dos seis pacientes que apresentaram alterações proliferativas, quatro $(66,7 \%)$ eram homens, cinco $(83,3 \%)$ tinham idade igual ou maior a 60 anos, quatro $(66,7 \%)$ não apresentavam sintomas dispépticos, três (50\%) estavam em uso de uma dose de menos de $40 \mathrm{mg} /$ dia e a outra metade estava em uso de uma dose igual ou maior de $40 \mathrm{mg} / \mathrm{dia}$, todos (100\%) usavam IBP por um tempo maior ou igual a 2 anos e cincno (83,3\%) não tinham o H. pylori presente na histopatologia.

As características dos seis indivíduos que apresentaram alterações proliferativas na mucosa oxíntica estão relacionadas na Tabela 4.

TABELA4-Características dos seis indivíduos que apresentaram alterações proliferativas na mucosa oxíntica 2009

\begin{tabular}{|c|c|c|c|}
\hline Características & & $n$ & $\%$ \\
\hline \multirow[t]{2}{*}{ Sexo } & Feminino & 2 & $(33,3 \%)$ \\
\hline & Masculino & 4 & $(66,7 \%)$ \\
\hline \multirow[t]{2}{*}{ Idade } & $<60$ anos & 1 & $(16,7 \%)$ \\
\hline & $\geq 60$ anos & 5 & $(83,3 \%)$ \\
\hline \multirow[t]{2}{*}{ Sintomas Dispépticos } & Presente & 2 & $(33,3 \%)$ \\
\hline & Ausente & 4 & $(66,7 \%)$ \\
\hline \multirow[t]{2}{*}{ Dose atual IBP } & $<40 \mathrm{mg} / \mathrm{dia}$ & 3 & $(50,0 \%)$ \\
\hline & $\geq 40 \mathrm{mg} / \mathrm{dia}$ & 3 & $(50,0 \%)$ \\
\hline \multirow[t]{2}{*}{ Tempo de uso IBP } & $<24$ meses & 0 & $(0,0 \%)$ \\
\hline & $\geq 24$ meses & 6 & $(100,0 \%)$ \\
\hline \multirow[t]{2}{*}{ H. pylori na histopatologia } & Presente & 1 & $(16,7 \%)$ \\
\hline & Ausente & 5 & $(83,3 \%)$ \\
\hline
\end{tabular}

$\mathrm{IBP}=$ inibidores de bomba de prótons $/ \mathrm{H}$. pylori $=$ Helicobacter pylori

Com a utilização do Qui Quadrado (considerando $5 \%$ de significância) pôde-se observar associação entre idade e alterações proliferativas da mucosa oxíntica, o mesmo não ocorrendo com as outras variáveis (Tabela 5).

O resultado da associação entre idade e alterações proliferativas da mucosa oxíntica está representado na Figura 1. 
TABELA 5 - Associação entre as variáveis

\begin{tabular}{lcc}
\hline Variáveis associadas & & $p$ \\
\hline Alterações proliferativas & Idade & $0,0050 * 0,01$ \\
\hline Alterações proliferativas & Dose atual IBP & 0,4155 \\
\hline Alterações proliferativas & Tempo de uso IBP & 0,0786 \\
\hline Alterações proliferativas & Sintomas dispépticos atuais & 0,1324 \\
Alterações proliferativas & Infecção H. pylori & 0,2396 \\
\hline
\end{tabular}

*Significância $p<0,05$ / IBP - inibidores de bomba de prótons / H. pylori Helicobacter pylori

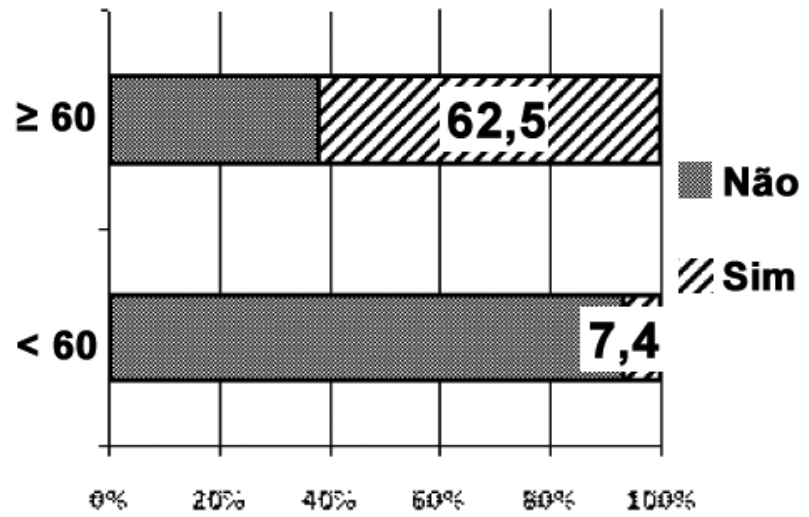

FIGURA 1 - Percentuais de indivíduos que apresentaram alterações proliferativas segundo idade maior ou igual a 60 anos e menor de 60 anos

\section{DISCUSSÃO}

Os IBP's são drogas bastante utilizadas na atualidade e demonstram excelente resultados no tratamento de doenças dispépticas. Há consenso de que eles podem ser prescritos de maneira segura e por longo período de tempo, entretanto é prudente que estudos constantes em grandes centros continuem sendo realizados.

O estudo deste grupo mostrou prevalência de $27,3 \%$ de alterações proliferativas da mucosa oxíntica em indivíduos em uso crônico de IBP's. Macroscopicamente, os pólipos fúndicos esporádicos foram às únicas alterações encontradas com prevalência de 9,1\%. Este achado foi maior que alguns relatos de literatura onde a prevalência variou entre $1,9 \% 10$ e 5,2\% 14 e foi menor que $16,3 \%{ }^{12}$, prevalência encontrada em estudo brasileiro. Esses foram encontrados em mucosa de fundo e corpo, poupando a mucosa antral, seguindo os relatos de estudos prévios ${ }^{8,12}$. Não houve achado de displasia nos pólipos encontrados neste grupo. Isto vai de encontro com descritos de literatura onde a displasia é achado raro e mais associado à síndromes polipóides ${ }^{17,9,13}$.

A alteração proliferativa microscópica achada foi exclusivamente a formação cística glandular, não sendo encontradas hiperplasia e protusão de células parietais como descritas em outros estudos ${ }^{2,11,14}$.
Encontrou-se relação entre idade $\geq 60$ anos e alterações proliferativas, a qual pode ser explicada pelo maior tempo de uso da droga, pois os cinco indivíduos que apresentaram alterações proliferativas já utilizavam inibidores de bomba de próton por tempo $\geq 24$ meses. Esta relação com idade nunca foi evidenciada na literatura.

Neste estudo, houve menor positividade do teste de urease comparando-se com a pesquisa histopatológica de $H$. pylori devido à diminuição da sensibilidade deste teste com o uso crônico de IBP's. Neste grupo estudado, um dos sujeitos que apresentou pólipo fúndico esporádico demonstrou infecção por H. pylori. Este fato não corrobora os relatos anteriores de que os pólipos teriam correlação negativa com a presença desta bactéria, 5,6,10,15,16.

O ponto forte deste estudo foi a seleção rigorosa de indivíduos que deveriam estar em uso crônico e contínuo de IBP, observando-se que a maioria dos indivíduos incluídos fazia uso a mais de um ano. Contudo, limitação aqui encontrada foi o número reduzido de participantes incluídos.

Mais estudos são necessários para esclarecer a importância clínica das alterações gástricas proliferativas ocasionadas com o uso crônico desta classe de medicamentos avaliando sintomatologia, evolução, risco de displasia, resolução e relação com fatores como presença do $H$. pylori.

O seguimento endoscópico de pólipos e outras alterações proliferativas relacionadas ao uso crônico de IBP's não está claramente estabelecida visto que a história natural destas alterações não está elucidada por completo.

Talvez, o manejo mais adequado para indivíduos que tenham alterações proliferativas como pólipos é a polipectomia dos maiores e suspensão dos IBP's devido sua possível associação com o surgimento desses pólipos.

Apesar do tratamento com IBP's por longo período ser considerado seguro atualmente, recomenda-se que sua utilização obedeça a indicações precisas e períodos pré-estabelecidos pelo médico assistente.

\section{CONCLUSÕES}

Ocorrem alterações proliferativas de mucosa oxíntica em indivíduos em uso crônico de inibidores de bomba de prótons. A associação entre idade e alterações proliferativas apresentou significância estatística.

\section{REFERÊNCIAS}

1. Bianchi LK, Burke CA, Bennett AE, Lopez R, Hasson H, Church JM. Fundic gland polyp displasia is common in familial adenomatous polyposis. Clin Gastroenterol Hepatol 2008; 6: 180-5. 
2. Cats $A$, Schenk BE, Blomena E, Roosedaal R, Lindeman J, Biemond I, Klinkenberg-Knol EC, Meuwissen SG, Kuipers EJ. Parietal cells protusions and fundic gland cysts during omeprazole maintenance treatment. Hum Pathol 2000; 31: 684-90.

3. Declich P, Ambrosiani L, Bellone S, Tavani E, Prada A, Bortoli A, Gozzini C. Fundic gland polyps under omeprazole treatment. Am J Clin Pathol 1999; 112: 576-7.

4. Declich P, Ambrosiani L, Grassini R, Tavani E, Bellone S, Bortoli A, Gozzini C, Prada A. Fundic gland polypus: a still elusive entity on the eve of the year 2000. Pol J Pathol 2000; 51: 3-8.

5. Declich P, Tavani E, Ferrera A, Caruso S, Bellone S. Sporadic fundic gland polyps: clinico-pathologic features and associate diseases. Pol J Pathol 2005; 56: 131-7.

6. el-Zimaity HM, Jackson FW, Graham DM. Fundic gland polyps developing during omeprazole therapy. Am J Gastroenterol 1997; 92: 1858-60.

7. Freeman HJ. Proton pump inhibitors and and emerging epidemic of gastric fundic gland polyposis. World J Gastroenterol 2008; 14 1318-20.

8. Huguet JM, Ruiz L, Ortí E, Luján M, Sempere J, Medina E. Poliposis gástrica secundaria a tratamiento com inhibidores de la bomba de protones. Gastroenterol Hepatol 2009; 32: 88-91.

9. Jalving M, Koornstra JJ, Gotz JM, van der Waaij LA, de Jong S, Zwart $\mathrm{N}$, Karrenbeld A, Kleibeuker JH. High grade dysplasia in sporadic fundic gland polyps: a case report and review of the literature. Eur J Gastroenterol Hepatol 2003; 15: 1229-33.
10. Jalving $M$, Koornstra JJ, Wesseling J, Boezen HM, de Jong $S$, Kleibeuker JH. Increased risk of fundic gland polyps during longterm proton pump inhibitor therapy. Aliment Pharmacol Ther 2006; 24: 1341-48.

11. Meuwissen SGM, Craanen ME, Kuipers EJ. Gastric mucosal morphological consequences of acid suppression: a balanced view. Best Practice \& Research Clinical Gastroenterology 2001; 15: $497-510$.

12. Morais DJ, Yamanaka A, Zeitune JMR, Andreollo NA. Gastric Polyps: a retrospective analysis of 26,000 digestive endoscopies. Arq Gastroenterol 2007; 44: 14-17.

13. Stolte M, Vieth M, Ebert MP. High-grade dysplasia in sporadic fundic gland polyps: clinically relevant or not? Eur J Gastroenterol Hepatol 2003; 15: 1153-6.

14. Vieth $M$, Stolte M. Fundic gland polyps are not induced by proton pump inhibitor therapy. Am J Clin Pathol 2001; 116: 716-720.

15. Watanabe N, Seno H, Nakajima T, Yazumi S, Miyamoto S, Matsumoto S, Itoh T, Kawanami C, Okasaki K, Chiba T. Regression of fundic gland polyps follwing acquisition of helicobacter pylori. Gut 2002; 51: 742-5.

16. Yamamoto A.; Ishiguro $H_{\text {.; }}$ Kondo $T$, Naruse $S$, Goto $H$. Low prevalence of helicobacter pylori infection in patients with fundic gland polyps. Nippon Rinsho 2005; ; 63: 621-4. 